\title{
Volume modelling for emerging manufacturing technologies
}

\author{
V CHANDRU and S MANOHAR \\ Department of Computer Science and Automation, Indian Institute of Science, \\ Bangalore 560012, India \\ e-mail: [chandru,manohar]@csa.iisc.ernet.in
}

\begin{abstract}
The next generation manufacturing technologies will draw on new developments in geometric modelling. Based on a comprehensive analysis of the desiderata of next generation geometric modellers, we present a critical review of the major modelling paradigms, namely, CSG, B-Rep, non-manifold, and voxel models. We present arguments to support the view that voxel-based modellers have attributes that make it the representation scheme of choice in meeting the emerging requirements of geometric modelling.
\end{abstract}

Keywords. Voxel modelling; virtual prototyping; layered manufacturing; rapid prototyping; reverse engineering.

\section{Introduction and motivation}

A confluence of three major technological revolutions is providing the impetus to the quantum changes in manufacturing technologies as we move into the next millennium.

(1) Information technology: Increased power of processors, decrease in the cost of memory, evolution and acceptance of the Internet and its rapid growth, the World Wide Web, multimedia and virtual reality technologies, distributed and parallel computing, growth of terrestrial, satellite, and broadcast communications, are some of the components of information technology that have a direct impact on manufacturing. For instance, the information superhighway with teleconferencing facilities will lead to large scale telecommuting of information workers. Computer-supported collaborative work will enable a geographically separated workforce to work as a team.

(2) Material technology: Several new materials called smart materials, materials with memory, composites with highly specialised functionality, low temperature superconducting materials, materials for fibre-optic communication, high efficiency solar energy converters, materials for solid-state batteries etc. have evolved.

(3) Process technology: Advances in VLSI technology have enabled the production of multi-million transistor chips and $256 \mathrm{Mbit}$ memories. The experience with VLSI technology has inspired two diametrically opposite process technologies, namely micro electro-mechanical systems (MEMS) and layered manufacturing technologies (LMT) 
(also referred to as rapid prototyping technologies, RPT). Both have their genesis in VLSI fabrication technologies and both require highly interdisciplinary research and development. Similar to VLSI technology, these two technologies depend critically on the development of new and powerful CAD tools.

(i) MEMS: In this technology, the sensors, the processors and the effectors can all be fabricated in the same process. An example is a commercially available radar, costing $\$ 5$ that can be fitted into automobiles to detect potential collisions with other automobiles. MEM systems promise to be the ultimate in mass production of electro-mechanical components, finished assemblies in millions of units at per unit costs of a few dollars.

(ii) RPT: A range of new processes variously called layered manufacturing, additive manufacturing and three-dimensional printing have emerged during the past few years (Burns 1993). These processes fabricate a component directly from the CAD description without the need for any additional fixtures, dies or tools. As opposed to the traditional approach of removing unwanted material from a block to arrive at the desired shape, these new processes build an object by selectively adding material where required by the geometric model. This technology promises to be the ultimate in customization, enabling the economical fabrication of one-of-akind finished assembly of complicated subunits.

In addition to the three technological streams the changing economic scenario is forcing a relook at manufacturing strategies. The rapid globalisation of the economy and the increasing competitiveness in the manufacturing sector has led to new paradigms like concurrent engineering, just-in-time manufacturing and business process reengineering. Product cycle times have come down from a few years to a few months and less. From concept to the customer, time required has been constantly shrinking and now we have reached a stage where custom products and low-volume high-custom products have begun to dictate competitiveness. Agile manufacturing is the term coined to reflect this ability to meet ever-changing product specifications. In areas like wearable computing (Finger et al 1996), where the associated technologies are constantly changing, completely new design philosophies have become essential.

The combined impact of these changes can be expected to drastically change manufacturing. Some of the extrapolated possibilities are conjectured below.

- Customer-in-the-loop manufacturing: For example, custom kitchens are designed by the customer using VR interfaces, built and delivered later at the customer's residence (Nomura et al 1992).

- Coin-operated manufacturing/shopping-mall manufacturing: The previous scenario refers to consumer durables. A similar quest for customization of consumer goods like shoes and personal accessories may result in compact manufacturing units being located in shopping malls, where the customer specifies the size (by placing the feet in a shoe mock-up that has built-in sensors) and waits while the shoe is fabricated.

- Just-in-place manufacturing: The next step is to sell economical manufacturing units to the customers so that the end products are fabricated just-in-time and just where it will 
be used. Examples are: kitchenware (spoons, forks, plates etc.) and toys. The customer uses highly interactive tools to design the objects and fabricates them in-house. The equipment has to be capable of making a range of shapes using a range of input raw material (fresh or recycled).

- Space-based manufacturing: As humans expand into space, by first building space stations and later moving to the planets, newer manufacturing technologies will be needed: flexible systems that occupy minimal volume and have minimum weight and are capable of fabricating a range of complex shapes will be required.

- Distributed product life-cycle management: Products will be conceived, designed, fabricated, marketed, maintained and upgraded by collaborative teams distributed geographically.

When we look at this exciting spectrum of possibilities, as well as the as-yet unthought of new processes and materials, it becomes very important to examine the tools we need to make them happen. At the fundamental level, any material object (mechanical, electromechanical) to be fabricated needs a model that forms the canvas on which the design process generates diverse paintings. Any such object is captured by a volume model. We use the term volume model since it is more general than the term solid model: a volume model can represent non-homogeneous objects (composites), flexible objects, and objects that are made of solid, liquid and amorphous material.

In this paper we examine the fundamentals of volume modelling for emerging manufacturing technologies. We first outline a set of requirements that a volume modelling paradigm should satisfy. This is followed in $\$ 2$ by a brief outline of existing modelling paradigms and their comparison based on our desiderata. In the final three sections we look in turn at the requirements of three major aspects of manufacturing, namely, virtual prototyping, physical prototyping and reverse engineering, and indicate how voxel modelling is uniquely suited to meet these requirements.

The major thrust of this paper is that voxel-based modelling has substantial unexploited advantages over other modelling paradigms.

\subsection{Desiderata for a volume model}

We present below a set of attributes that can provide a comprehensive characterization of a representation scheme for volume modelling. We have grouped these attributes to reflect the three phases in the lifecycle of mechanical parts:

- Model creation and maintenance

- renderability

- morphological dexterity

- heterogeneity

- editability

- brevity 
- Model analysis

- analysability

- Model fabrication

- reconstructibility

- physical realizability

- accuracy

\subsection{Renderability}

This captures the ease with which the volume model can be rendered. Parameters to be considered are the complexity of the rendering computation and the quality of the rendered images as well as the ability to render the model at various levels of quality. For interactive modelling, the ability to rapidly preview the model, interact dynamically with the model as well as get high quality presentation images, are all essential.

\subsection{Morphological dexterity}

The ability to represent a variety of shapes is an important aspect of any volume modeller. Traditionally solid modelling has focused on representing shapes that are restricted to manifold topologies. However, the importance of non-manifold representations has been recognized (XOX Corporation 1995).

The morphological dexterity attribute is aimed at capturing the complexity of shapes that can be represented and the ease with which complex shape modifications can be achieved on the given volume representation.

\subsection{Heterogeneity}

Traditional CAD tools available today implicitly assume that the part being designed is to be fabricated using a single homogeneous material. A few niche areas like aircraft/spacecraft use specialised CAD tools to model composites. The need for general CAD tools to handle composites will be realized as an increasing range of products are fabricated with composites.

The heterogeneity attribute will evaluate the ability of a representation to capture inhomogeneities of the following types: use of different materials, use of different densities of the same material across the cross-section of the solid, use of hollow structures (example: honeycomb) and integrated electromechanical components.

\subsection{Accuracy}

When evaluating a volume representation scheme with reference to the accuracy of the representation the following factors have to be considered:

The accuracy with which the model captures the intended shape of the part is of primary importance. Of equal importance is the ease and accuracy of specification of tolerances consistent with the ideal design (Requicha 1984). 


\subsection{Editability}

The central purpose of a computer-based modelling paradigm is to empower the user to effect changes to the model. We intend the broad term "editability" to encompass both local changes as well as global changes in the model. Examples of global changes are the scaling or shearing of the entire part in a single operation. A less obvious, but equally important aspect of editability is the system's ability to allow the user to make such changes (both global and local) interactively.

\subsection{Brevity}

Regardless of how inexpensive available memory has become, the requirements of users have grown to make the compactness of the representation a critical issue. We intend the brevity attribute to reflect the size of the model, the amenability of the representation to various compression schemes and the ease with which modelling operations can be performed on the compressed representation.

\subsection{Analysability}

Analysability denotes the amenability of the representation to the following tasks in the design cycle: Computation/extraction of physical parameters from the model to validate against specifications, functional analysis of the model (using finite element methods for example), and the visualization of the results of the analysis.

\subsection{Reconstructibility}

Reconstructibility is the attribute that qualifies the ease with which volume representation can be obtained from a physical part, using any one of several measurement modalities (e.g. range sensors, CAT scans, MRI scans etc.), or from a different volume representation of the same part.

\subsection{Physical realisability}

The usefulness of any volume representation scheme is considerably enhanced if a volume model can be used to drive a fabrication process. In addition, the model may be used to determine various attributes of alternate fabrication processes. An example of such an attribute is the time to machine a part. Physical realisability is the attribute of a volume representation that denotes these capabilities.

\section{Volume modelling: The state of the art}

In this paper we examine four dominant modelling schemes, namely the boundary-representation (B-rep), non-manifold, constructive solid geometry (CSG), and voxel modelling schemes. Figure 1 (Hoffmann 1989) shows simple examples for each of the above schemes. 
(a)

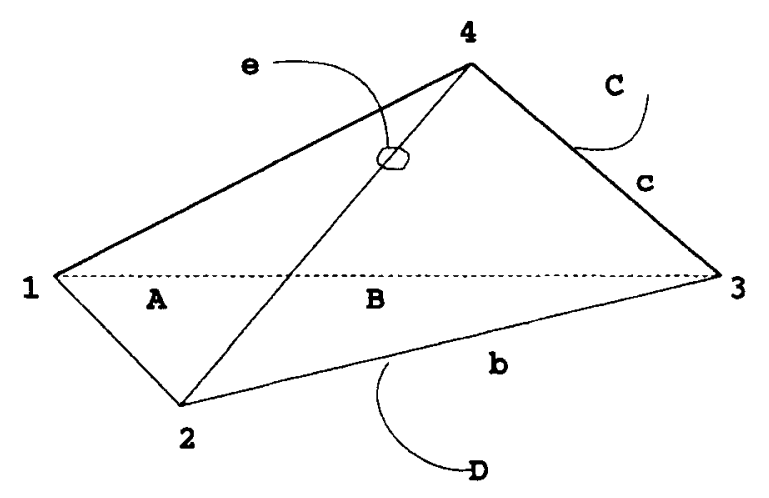

\begin{tabular}{|c|c|c|c|c|c|c|c|c|}
\hline Edge & \multicolumn{2}{|c|}{$\begin{array}{l}\text { Vertices } \\
\text { From To }\end{array}$} & \multicolumn{2}{|c|}{$\begin{array}{l}\text { Faces } \\
\text { Left Right }\end{array}$} & \multicolumn{2}{|c|}{$\begin{array}{l}\text { Clockwise } \\
\text { Pred Succ }\end{array}$} & \multicolumn{2}{|c|}{$\begin{array}{l}\text { Counter } \\
\text { Clockwise } \\
\text { Pred Succ }\end{array}$} \\
\hline $\mathbf{a}$ & 1 & 2 & A & D & d & $\theta$ & $f$ & b \\
\hline$b$ & 2 & 3 & B & D & $\theta$ & c & $\mathbf{a}$ & $f$ \\
\hline $\mathbf{f}$ & 3 & 1 & C & D & c & $a$ & b & a \\
\hline c & 3 & 4 & B & C & b & e & $\mathbf{f}$ & d \\
\hline d & 1 & 4 & C & A & $\mathbf{f}$ & c & $\mathbf{a}$ & e \\
\hline$\theta$ & 2 & 4 & A & B & a & d & b & c \\
\hline
\end{tabular}

Figure 1. (a) Boundary-representation (B-rep) model.

The Boundary-Representation (B-Rep) scheme is a natural extension of the topological description of rigid solids, using complex data structures to represent vertices, edges and faces of a solid along with the topological constraints (such as adjacencies) and an elaborate system of pointers. A delicate balance has to be struck in B-Rep modellers to balance between efficiency of modelling operations and fast response to interrogations of the model usually implemented via redundant access paths. A thorny issue for B-Reps has remained the issue of robustness. Geometric computations and topological constraints in the framework of a redundant data structure can often lead to internal inconsistencies in the representation and to system crashes. Commercially available B-Rep systems include ROMULUS, ACIS, CATIA, EUCLID and GEOMOD. However, the system that 
(b)

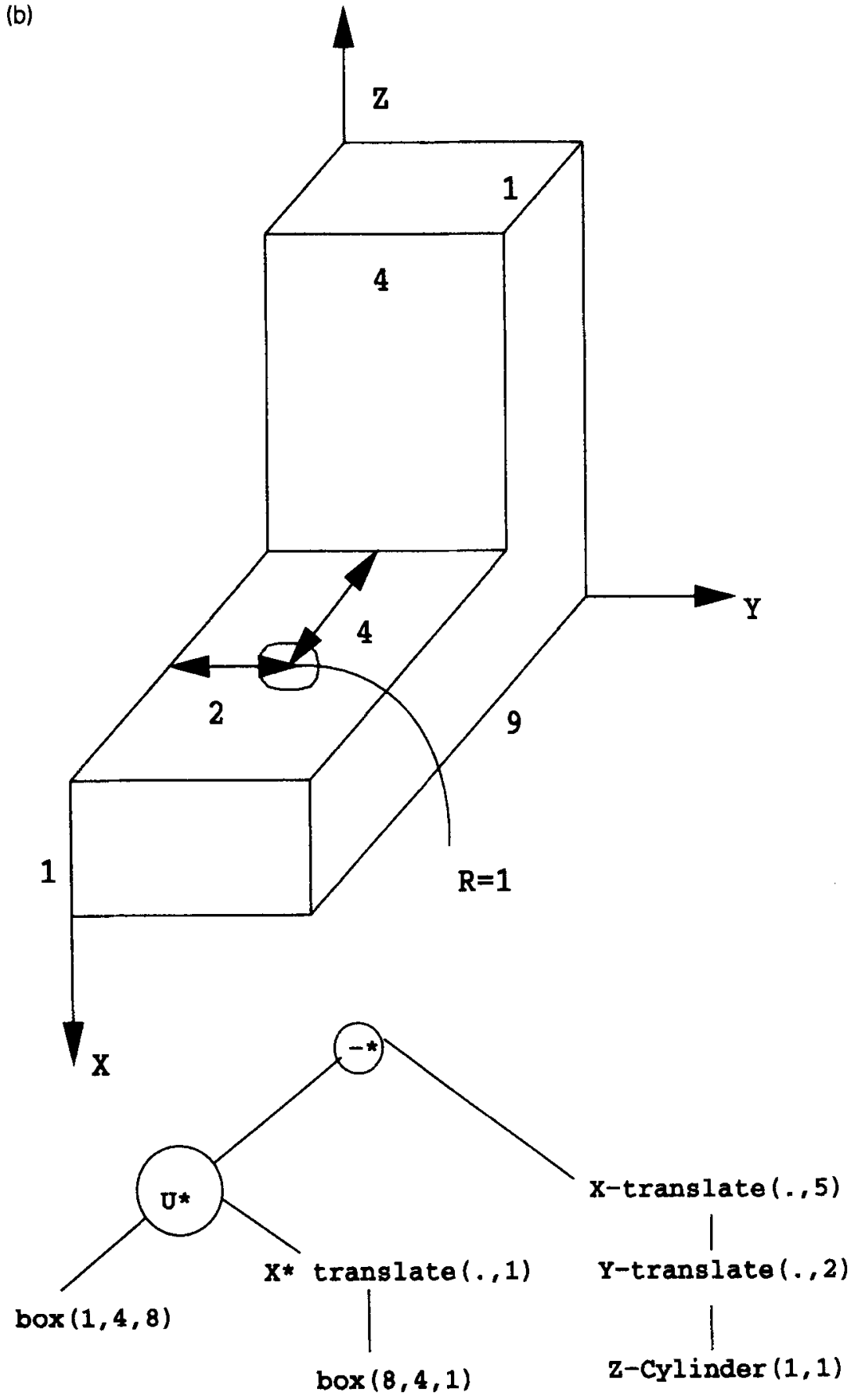

Figure 1. (b) Constructive solid geometry (CSG) model. 


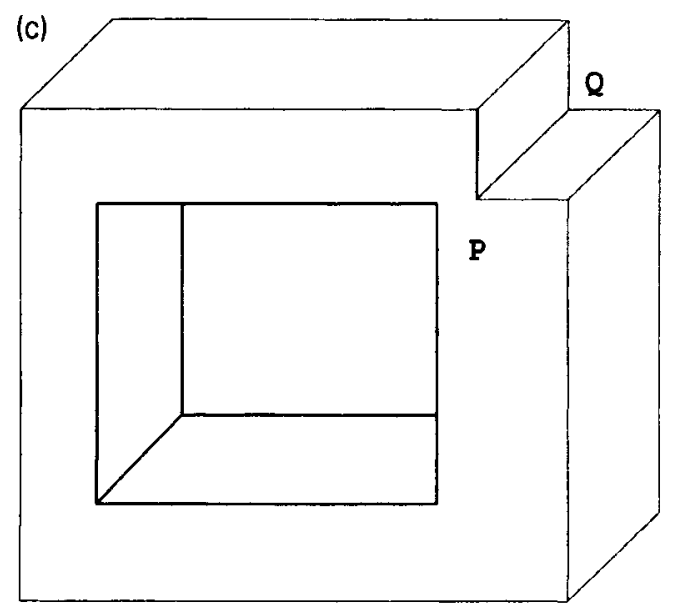

Figure 1. (c) Non-manifold model.

popularized B-Rep modellers was the BUILD modeller, originally developed by IC Braid at Cambridge in 1979.

Constructive Solid Geometry (CSG) Modellers build representations of complex rigid objects by applying unary and binary operators such as set union, intersection, difference to elementary volumetric primitives such as blocks, cylinders, cones, spheres and tori. The representation is usually schematically shown as a tree with the root representing the solid and the leaves representing the primitives. The CSG modelling scheme was proposed and implemented (PADL-2) by a dedicated group of researchers at the University of Rochester (headed by H B Voelcker and A A G Requicha) in the early 1980's. The CSG representation is remarkable for its simplicity and succinctness and has therefore played an important role in the design of user interfaces for solid modellers. However, it is inadequate on some counts such as ease of rendering and analysis.

The Non-Manifold Representation was developed as an attempt to meet the need for a scheme in which the transition between 1D, 2D and 3D entities can be seamlessly executed. Dangling edges and faces, solids touching at vertices and edges and in the middle of faces, and other such irregular geometric constructs have to be valid in such a representation. Modellers built around such representations would be closed under general Boolean set operators and general sweep operators. Weiler (1988) proposed a radial edge data structure that could support such a representation. The approach that Weiler took was to extend the traditional B-Rep structure to admit non-manifold representations. SHAPES (XOX Corporation 1995) is a commercial non-manifold modeller developed and maintained by the XOX Corporation.

Voxel modellers, Spatial Occupancy Enumeration or Cellular Modellers use a very simple representation scheme. A volume of space (containing the object) is divided into a large number of cuboidal cells. The system then labels each cell as to whether or not it is occupied by material. The obvious data structure for this representation is a 3D array of 0 's and 1's. More sophisticated data structures based on quadtree and octree representations have been implemented in the popular modellers TIPS-1 (built by Okino and others at Hokkaido University) and INSIGHT (a molecular modelling system built by Phoenix Data Systems). Pratt (1994) in his review of solid modelling, states that,“... most 
solid modelers based on a purely cellular approach are still in the research domain. This appears to be because a satisfactory trade-off has yet to be found between the requirement for accurate boundary resolution needed in engineering applications and the very large storage requirements that this implies."

Requicha (1980) published a seminal study of solid modelling representation schemes. A more recent update of this work is by Pratt (1994). The interested reader is directed to these papers for more details on the individual schemes. The above studies focus on the comparison of the B-Rep and CSG solid modelling schemes, while dismissing the voxel-based modelling schemes as being impractical.

In this paper we re-evaluate these three schemes with reference to the desiderata listed below in table 1 . As mentioned above, the non-manifold modelling paradigm evolved as an extension of the B-Rep representation scheme. However, in principle, non-manifold modellers need not follow only this line of development. Hence we evaluate non-manifold representation schemes as separate from B-Rep of solids as also indicated in table 1.

A representation that flags geometric features of a part that helps identify manufacturing processes that match with a particular feature will enjoy tremendous increases in efficiency of the CAD system built around it. Such hierarchical representation schemes have come to be called feature-based. The need for them grew out of the fact that B-Rep and CSG representations are too low-level for a designer to work with. Feature-based schemes are thus an expedient interface between CAD systems today and analysis or manufacturing applications (Rossignac 1990). We do not consider this scheme in our comparative study of volume representations since feature-based models exist at a higher level and can, in principle, be equally well-integrated with any of the B-Rep, CSG, Voxel or Non-Manifold volume representation schemes.

Table 1 summarizes our evaluation of the various schemes for volume modelling via a rating of each scheme on each of the ten attributes in our list of desiderata. The following are brief remarks on the ratings given in the table. The rating of voxel models will be discussed in some detail in the sections that follow.

The boundary representation scores high on renderability, morphological dexterity, accuracy, editability (local and global), analysability, reconstructibility and physical realisability. The detailed nature of this representation, with explicit access of vertex, edge and face components, permits efficient data structures and computations on B-Reps. The ability

Table 1. Attributes of the dominant modelling schemes.

\begin{tabular}{lllll}
\hline Attribute & B-Rep & CSG & Non-manifold & Voxel \\
\hline Renderability & Easy & Hard & Easy & Hard \\
Morph. dexterity & Good & Poor & Good & Excellent \\
Heterogeneity & Impossible & Hard & Hard & Easy \\
Accuracy & Excellent & Poor & Excellent & Poor \\
Editability (local) & Good & Poor & Good & Excellent \\
Editability (global) & Good & Poor & Excellent & Poor \\
Brevity & Fair & Excellent & Fair & Poor \\
Analysability & Good & Poor & Good & Good \\
Reconstructibility & Good & Poor & Good & Excellent \\
Physical realizability & Easy & Hard & Hard & Easy \\
\hline
\end{tabular}


in principle to accommodate free-form bounding surfaces explains the high accuracy and morphological dexterity of this representation. On the other hand, the B-Rep scheme is verbose and has no provision to access volume elements in the interior of objects, and hence this representation scores low on the counts of brevity and heterogeneity.

Constructive solid geometry representations fare somewhat poorly in our evaluation. The only count on which we favour this representation is its brevity. This representation played an important role in the early development of solid modelling because it permitted approximate representation of complex shapes via regularized Boolean operations on simple primitives. However, the indirect and implicit form of this representation makes it cumbersome for interrogations that involve structure of the model.

Non-manifold models have even more detail explicitly given in their representation than B-Rep models. Hence they enjoy all the advantages of B-Rep models except on the count of physical realisability. The non-manifold representation allows features such as dangling edges and faces which are hard to fabricate. We rate non-manifold representations a little higher than B-Reps on global editability because of access to volume elements. On the negative side, non-manifold models suffer from low ratings on brevity and heterogeneity just as B-Reps do. In principle, because of access to volume elements, it is possible to work some heterogeneity into non-manifold models but this seems to involve considerable overheads.

\section{Voxel modelling in emerging manufacturing technologies}

In this section we focus on the attributes in which the Voxel methods outperform the other modelling schemes as shown in table 1 . We believe that it is this set of attributes which can be exploited to tackle the challenging problems encountered by newer manufacturing technologies.

\subsection{Voxel modelling for virtual prototyping}

"Virtual prototyping is the process of design analysis, simulation and testing of a product within the computer and use of the results to refine the product before making a physical prototype" (Kumar et al 1996).

The excellent morphological dexterity of voxel models combined with their excellent local editability imply that CAD tools built using voxel models will allow arbitrary shape manipulation and sculpting. Such expectations have been fulfilled by interactive voxel modelling tools currently under development (Ravi 1996; Sethia 1996).

In VoxLab (Ravi 1996), it is possible to edit objects at the voxel level as well as use global operations to modify the volume structure of the object.

Interactive sculpting is the process by which a designer can impose arbitrary free-form shape changes on the object being designed. Considerable work has been done in the field of surface-based sculpting, which can be broadly classified into space deformation and surface fitting. Terzopoulos \& Qin $(1994,1995)$ have developed dynamic NURBS and dynamic triangular B-Spline models which are physics-based models. These models are used for interactively sculpting curves and surfaces by applying simulated forces and local and global shape constraints. Pasko \& Savchenko (1995) have approached the problem of interactive sculpting as the local deformation of a constructive solid by a set of arbitrary points 
that belong to the resultant surface. Rappoport et al (1995) have developed a method for modelling an object which is composed of several tensor product solids, while preserving the desired volume of each primitive. Borrel \& Rappoport (1994) have proposed a model for producing controlled spatial deformations. In their model, the user defines a set of control points, giving a desired displacement, and radius of influence of each. Each constraint point, determines a local B-Spline basis function centred at the control point, and falling to zero for points beyond the radius. Rappoport et al (1994) have introduced the concept of soft constraints which need not be met exactly, thus increasing the design space. Finally, McCracken \& Joy (1996) have proposed a new free form deformation technique that generalizes previous methods by allowing 3D deformation lattices of arbitrary topology.

The main drawbacks of these methods are that they are object-complexity sensitive, they do not operate on the object being sculpted directly, and it is very hard to modify the topology of the object being designed.

Voxel-based sculpting is proving its promise in our on-going work. We use Minkowski operations to sculpt voxel blocks using tools of arbitrary shape which are themselves voxel models (Sethia 1996). An object created by such sculpting operations is shown in figure 2 .
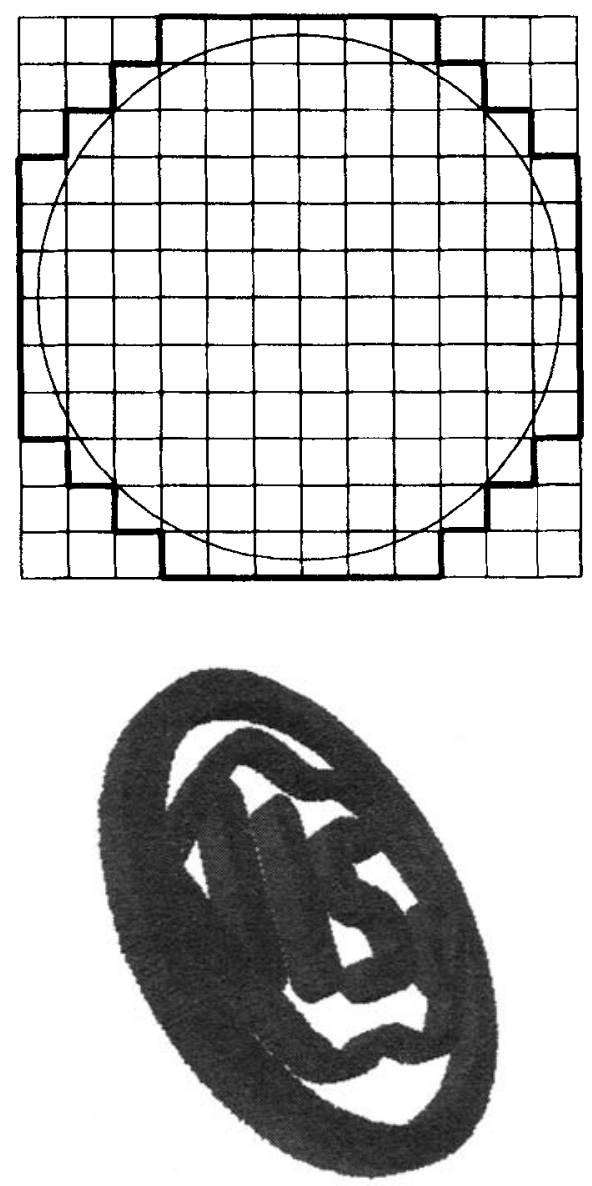

Figure 2. Spatial enumeration model. 
Mechanical analysis is an integral part of the design process since the physical properties of the parts determine if a particular design is viable or not. Traditional CAD tools for analysis use the finite element method (FEM) to compute various material properties like structural strength, thermal conductivity etc. on the geometric representation of the part. The FEM methodology is essentially a technique for computing discrete approximations of continuous physical quantities. For B-Rep or CSG tree models the discretisation required by FEM is realized by tetrahedralisation of the design volume. A major computational issue in this context is the maintenance of non-degenerate tetrahedralisation (ensuring connectivity, eliminating hanging edges etc.). These problems do not arise in a voxel model since the basic unit of a voxel model is a volume-filling solid, namely, the voxel. Thus, accurate FEM discretisations can be easily extracted from a voxel-model for analysis.

The visualisation of the results of the analysis is facilitated by volume models: direct volume rendering techniques can be used to render the results of the analysis volumetrically superimposed on the voxel-model. Interactive volume visualisation tools allow the user to view the results along any cutting plane of the model, or as a composite whole.

Several problems that are difficult using B-Rep or CSG or non-manifold approaches have a simple solution using voxel models. These include estimation of mass properties, interference detection, tolerancing and implementation of CSG operations.

Tolerancing is an important aspect of mechanical design (see ElMaraghy et al 1994, for example). A voxel-based modeller can handle this aspect of the design as follows. The intersection of two objects due to the variation in tolerance is visualized as a $3 \mathrm{D}$ interference volume itself. The interference volume is a measure of the extent of interference, and therefore a measure of the "out-of-tolerance" or deviation of the object. The tolerance values can be reassigned, and a decreasing volume of intersection indicates a correct trend of tolerance alteration.

The use of interference volumes can be extended to solve the problem of assemblability and disassemblability testing. Other modelling schemes require complex geometric intersection algorithms and may also suffer from numerical inaccuracies. With voxel models, the interference can be precisely quantified and accurately located.

Combining the good analysability of voxel models with global and local editability, it is possible to design objects with excellent shape optimization. A naive approach is the following: Map the results of the engineering analysis to the voxel model. Use a global filter that removes voxels that do not have any load on them, after ensuring that those voxels are not essential for maintaining the structural integrity of the part. An example of a shape-optimizing structure is a honeycomb mesh that can replace solid interiors at locations on the part that are not subjected to any loads. This will reduce the overall weight of the object as well as result in material saving. The morphological dexterity of voxel models permits more sophisticated optimizations that will be impossible on B-Rep or CSG models.

\subsection{Voxel modelling for physical prototyping}

In spite of the tremendous strides made in virtual prototyping technologies, physical prototypes are indispensable in the concurrent engineering process. Hence attention is 
being directed at making these physical prototypes as rapidly as possible. Traditional prototyping processes range from wooden mockups, casting from custom patterns, machining, and the use of sophisticated NC machines that are directly programmed from the CAD model of the part. Rapid prototyping, the ability to produce a prototype very early in the design cycle, even before the tooling for the manufacture of the component has been designed, has emerged as a critical technological component of concurrent engineering. Recently, several new manufacturing technologies that support rapid prototyping have become available both in research laboratories and in the commercial marketplace. These technologies are variously called layered manufacturing, additive manufacturing, and stereolithography (Jacobs 1992). These manufacturing techniques allow a part, or a prototype, or a tool to be built by the gradual addition of material in a controlled way. In contrast, traditional manufacturing methods depend on the the removal of material (milling, turning etc.) or on the deformation of material (casting, moulding etc). The field of rapid prototyping is making rapid strides (Burns 1993).

RPTs provide the means to manufacture parts that cannot be realized by conventional methods (Kruth 1992). Arbitrary shapes, composite materials, and complicated geometries can be as easily manufactured as simpler geometries. In order to exploit these capabilities, the modelling schemes used by CAD tools to design parts should be capable of handling a range of shapes including non-manifold objects. Thus, non-manifold schemes (XOX Corporation 1995) and voxel-modelling are the only schemes that have this capability.

However, for converting a virtual prototype into a physical prototype using RPT technology, the current practice is to convert all models into the stereolithography format (STL format) that has emerged as the de facto standard. The model in STL format, basically a 3D triangulation, is then input to a slicing process (that is unique to each of the layered manufacturing equipment) that generates a sequence of slices that drive the material addition process.

These two steps, namely, converting into a STL format and the slicing of the STL format introduce changes in the model that are beyond the control of the part designer.

Using a voxel-based modelling scheme it is possible to completely eliminate the above two steps and instead drive the fabrication process directly. This is facilitated by the close correspondence between voxel models and the fabrication processes.

The current range of rapid prototyping machines can be broadly classified into two major categories based on the way in which material is added to an object under construction.

- Parallel/image-based: The most prominent system under this class is the Solider MPM system of Cubital, which is a commercially available system. A well-known research system under this category is the MD* (Weiss \& Prinz 1991). In these systems, successive layers of the component under fabrication are created by the use of masks that either allow a light source to solidify a photopolymer under the exposed regions, or spray material on the exposed parts of the mask. The advantage of this approach is that the time for creating a complete layer does not depend on its geometric complexity. Each mask is simply a slice of the object, and can be thought of as the image of the object's cross-section.

- Sequential/vector-based: The pioneering rapid prototyping systems, the SLA machines from 3D Systems, as well as commercial systems from Stratasys and Helisys fall under 
this category. In these systems, a layer is formed by the sequential formation (deposition, or solidification) of the contours in the object's cross-section. Hatching or filling-in operation is needed to obtain solid interiors.

Some RPTs, still in the research stage, like the ballistic particle manufacturing, and the beam interference solidification process, create parts by solidifying individual voxels.

Even some layered manufacturing techniques like stereolithography and selective laser sintering use point-by-point solidification to grow each layer. Thus these processes work one level below the layer-by-layer manufacturing techniques and this involves the conversion of layer information to voxel information, usually performed by the manufacturing equipment itself.

At every stage in the design process, the description of the part is directly the description required by the manufacturing process. Thus it is easy to evaluate the design with respect to its manufacturability.

Several current and proposed RPTs generate parts by adding material one layer at a time. The MD* process (Weiss \& Prinz 1991) uses this approach using photographic masks and a vapour deposition process that is very similar to the technology used for VLSI fabrication. However, the input to the manufacturing device is currently obtained by a postprocessing step after the design of the part is completed. This step involves the generation of slices of the finished part, the number of slices generated depending on the layer resolution of the RPT. The hardware itself is neutral to the CAD tools or primitives that have been used in the design of the part. However, the use of traditional CAD tools that use either B-Rep or CSG as the basis will imply that the postprocessing step is left to deal with problems like the proper orientation of the part before slicing for optimal manufacturability (minimum use of support material, protection of the structural integrity of the part at every stage of the growth process etc.).

STL (Stereolithography list) was developed by 3D System for their stereolithography machine and has become a de-facto standard input format for several RPTs (Jacobs 1992). This PHIGS-based format approximates the part's inner and outer surfaces by a set of triangular plane patches. Each patch is described by its three vertices and a normal vector pointing out of the material.

Though it is possible to generate STL from voxel models to conform to existing RPTs, the voxel models permit generation of outputs in formats more closely matched to the target RPT. For instance, if the RPT uses a layer-by-layer approach, the voxel-based modeller can output the layer information after taking into account several process-planning steps, even during the design phase. This will eliminate the need for any processing by the RPT.

Composites: Research in LM technologies will make it feasible to fabricate components with several constituent materials that are physically located to ensure optimum performance. The extrusion process used by the Fused deposition modelling (FDM) of Stratasys (Burns 1993) can use polymers as well as metals of low melting point like tin. The MD* process of CMU uses a robotic arm to spray different metals over masks. It is very likely that in the near future, LM technology will mature to a point where multiple materials can be used to fabricate a single component.

The ease with which heterogeneity can be handled by voxel models enables designers to create composites using the same set of $\mathrm{CAD}$ tools that are used for homogeneous objects. 
Conventional design tools are not oriented to the design of composite objects. Specialized tools are used in areas like the aircraft industry where composite materials play a major role.

A voxel-based modeller can ultimately provide the capability to design a composite object with materials selectively placed at individual voxels. There is no need to compute the complex geometries of the interleaved materials since, for fabrication, each slice of the voxel buffer can be directly read out and several masks per layer can be created to deposit the different materials. Such capabilities will be indispensable as the technology of microelectromechanical systems (see Senturia et al 1992, for example) matures: A voxel will then be of molecular dimensions.

Inspection of the fabricated part to ensure its compliance with specifications is an important aspect of manufacturing. A voxel-based approach has been successfully used to implement an on-line automated visual inspection system (Tarbox \& Gottschlich 1995).

Fabrication of components with embedded electronics will be an increasingly common requirement. The design of wearable computers is one such example that requires rapid product development (due to the rate of change of the component technologies) and prototyping. A simple approach that exploits volume models in combination with layered manufacturing technology is as follows: Volume-scan the electronic subunit, combine with a voxel model to give an integrated model of the component and insert the electronic unit at a suitable time during fabrication. Implementation of this basic process requires advances in both $\mathrm{CAD}$ tools and layered manufacturing tools.

Chandru et al (1995) elaborate on the above advantages of using voxel models for rapid prototyping.

\subsection{Voxel modelling for reverse engineering}

Voxel models have excellent reconstructibility. This implies the following.

- It is easy to obtain the voxel model from a physical part. In practice, CAT and MRI scans directly give a three-dimensional voxel array of the model while range sensors or 3D scanners provide a cloud of points from which a voxel model can be obtained.

- It is easy to convert models from other representations to voxel models. This can be achieved using a class of algorithms called voxelisation algorithms (Wang \& Kaufman 1993) that convert geometric primitives into an array of voxels.

Reverse engineering is the process of construction of a CAD model from a physical part. Measurements are made on the physical prototype (typically by scanning) and these are then processed to obtain the CAD model. Thus voxel models are ideally suited to support reverse engineering.

In addition to the traditional reverse engineering task of obtaining a computer model from a physical prototype, the voxel-based approach leads to other possibilities. Reverse engineering in conjunction with the voxel-based modelling and analysis provides a means of reengineering a part from a sample physical model.

We are investigating the use of such reverse engineering in conjunction with rapid prototyping equipment to provide a platform for rapid product innovation and design (Chandru et al 1996). In this approach, in contrast to the prevalent use of rapid prototyping 
to validate the reverse engineered CAD models, we are proposing the use of reverse engineering to estimate errors in the RPT process and correct them using voxel-based modelling and volume-error measures.

\section{Research issues in voxel modelling}

Table 1 lists some attributes for which voxel models compare poorly with the other models. These are precisely the attributes that have been the bottlenecks to the widespread use of voxel models. In this section we address each of these attributes and indicate research directions aimed at overcoming the limitations.

Rendering: Current graphics systems are predominantly polygon-based. Recent desktop workstations, even at the entry level, are capable of rendering close to a million triangles per second. Similar hardware advances are yet to take place for volume rendering and hence rendering of reasonable voxel volumes (say $512 \times 512 \times 512$ ) takes a few seconds. However, various ways to speed up volume rendering are being vigorously pursued. These include the use of texture mapping hardware to perform back-to-front composition of volume slices (Fraser 1994), the development of special-purpose hardware for volume rendering (Pfister $e$ t al 1995), and the use of distributed and shared memory workstations.

Global editing: Voxel modelling has excellent local editability and certain global operations (analogous to filtering) that alter the entire model can be implemented with ease. However, they suffer from a lack of information about the component structures of an object. For instance, a voxel model of a table will allow one to edit the sharp corners into rounded corners, but it will be difficult to replace the four legs of the table with legs of different shape. This is because there is no notion of a substructure in the basic voxel models.

Research into hierarchical models that permit such substructuring, without disturbing the other advantages of voxel models may be thought of as feature-based modelling using the voxel as the basis. This appears to be a fruitful research direction.

Brevity: A serious practical problem with voxel models is their poor brevity. A regular voxel array to represent a component with a $5 \mathrm{~cm}$ bounding box will require a voxel resolution of about $400^{3}$ to realize a physical part with an accuracy of 125 microns.

Clearly, this is another reason for hierarchical and adaptive representations. Octrees, BSP trees and similar data structures combined with algorithms that effectively operate on these structures is a critical area of research. Another facet is that of compression algorithms and algorithms that operate on compressed data structures.

Accuracy: The accuracy of voxel models is inversely related to the brevity of the representation. Since by definition a voxel representation is a discretization of continuous geometric entities, the accuracy of the representation will be poorer when compared to CSG or B-Rep. Aliasing errors due to the discretization process will be present. However, the redeeming aspect of voxel models is the possibility of exact quantification of the error as well as user control of the error. Research into the application of anti-aliasing techniques of computer graphics to voxel models and the use of volumetric error measures is a promising area of research (Prakash \& Manohar 1995). 


\section{References}

Borrel P, Rappoport A 1994 Simple constrained deformations for geometric modelling and interactive design. ACM Trans. Graphics 18: 137-155

Burns M 1993 Automated fabrication (Englewood Cliffs, NJ: Prentice Hall)

Chandru V, Gurumoorthy B, Manohar S 1996 Integrated modelling and reverse engineering for layered manufacturing. Technical Report, CSA-IISc-1996-02, Indian Institute of Science, Bangalore

Chandru V, Manohar S, Prakash C E 1995 Voxel-based modelling for layered manufacturing. IEEE Comput. Graphics Appl. 42-47

ElMaraghy W H, Valluri S R, Skubnik S M, Surry P D 1994 Intersection volumes and surface areas of cylinders for geometrical modelling and tolerancing. Computer-Aided Design 26: 29-45

Fraser R 1994 Interactive volume rendering using advanced graphics architectures. SGI Dev. News December

Finger S et al 1996 Rapid design and manufacture of wearable computers. Commun. ACM 39(2): $63-70$

Hoffmann C M 1989 Geometric and solid modelling: An introduction (San Mateo, CA: MorganKaufmann)

Jacobs P F 1992 Rapid prototyping and manufacturing - Fundamentals of stereolithography. (Dearborn, MI: Society of Manufacturing Engineers)

Kruth J P 1992 New manufacturing techniques for rapid prototyping and concurrent engineering. Manufacturing in the era of concurrent engineering (eds) G Halevi, R D Weiel (New York: Elsevier Science)

Kumar V, Bajcsy R, Harwin N, Harker P 1996 Rapid design and prototyping of customized rehabilitation aids. Commun. ACM 39(2): 55-62

McCracken R, Joy K I 1996 Free-form deformations with lattices of arbitrary topology. Computer Graphics Proceedings, SIGGRAPH96, pp 51-60

Nomura J et al 1992 Virtual space decision support systems and its application to consumer showrooms. Matsushita whitepaper

Pasko A A, Savchenko V V 1995 Algebraic sims for deformation of constructive solids. Third symposium on solid modelling and applications, pp 403-408

Pfister H, Kaufman A, Wessels T 1995 A scalable architecture for real-time volume rendering. Proceedings of the Eurographics Workshop on Hardware, Masstricht, pp 123-130

Prakash C E, Manohar S 1995 Error measures and 3D Anti-aliasing for voxel data. Proceedings of Pacific Graphics 95 (Seoul: World Scientific)

Pratt M J 1994 Solid modelling - Survey and current research issues

Rappoport A, Hel-Or Y, Werman M 1994 Interactive design of smooth objects with probabilistic point constraints. ACM Trans. Graphics 18(2): 156-176

Rappoport A, Sheffer A, Bercovier M 1995 Volume preserving free form solids. Third symposium on solid modelling and applications, pp 361-372

Ravi N 1996 VoxLab: An interactive toolkit for voxel-based modelling. Masters Project Report, Department of Computer Science and Automation, Indian Institute of Science, Bangalore

Requicha A A G 1980 Representation of rigid solids - Theory, methods and systems. Comput. Surv. 12: 437-464

Requicha A A G 1984 Representation of tolerances in solid modelling: issues and alternative approaches. Solid modelling by computers (New York: Plenum)

Rossignac J R 1990 Issues on feature-based editing and interrogation of solid models. Comput. Graphics 14: 149-172 
Senturia S D et al 1992 A computer-aided design system for microelectromechanical systems (memcad). J. Microelectromech. Syst. 1(1): 3-13

Sethia S U 1996 Interactive volume sculpting. Masters project report, Department of Computer Science, Indian Institute of Science, Bangalore

Terzopoulos D, Qin H 1994 Dynamic NURBS with geometric constraints for interactive sculpting. ACM Trans. Graphics 18(2): 103-136

Terzopoulos D, Qin H 1995 Dynamic manipulation of triangular B-splines. Third symposium on solid modelling and applications, pp 281-291

Tarbox G H, Gottschlich S N 1995 IVIS: An integrated volumetric inspection system. Comput. Vision Image Understanding 61: 430-444

Wang S W, Kaufman A 1993 Volume sampled voxelisation of geometric primitives. Proc. of IEEE Visualization 93 (New York: IEEE Press)

Weiler K J 1988 The radial edge structure: A topological representation for non-manifold geometric boundary modelling. Geometric modelling for CAD applications (ed.) M J Wozny et al (Amsterdam: North-Holland)

Weiss L E, Prinz F 1991 A framework for thermal spray shape decomposition in the MD* system. Proc. of 1991 Solid Freeform Fabrication Symposium

XOX Corporation 1995 SHAPES microtopology, a system for modelling mesh geometries. XOX Corporation, 1450 Energy Park Drive, Suite 120, St. Paul, MN 55108 